Draft VERSion OCTOBER 23, 2018

Preprint typeset using $\mathrm{LAT}_{\mathrm{E}} \mathrm{X}$ style emulateapj v. 20/04/00

\title{
THE TYPE IA SUPERNOVA WIDTH-LUMINOSITY RELATION
}

\author{
Philip A. Pinto ${ }^{1}$ \\ Steward Observatory, The University of Arizona, Tucson, AZ 85721 USA \\ AND \\ Ronald G. EASTMAN ${ }^{2}$ \\ Lawrence Livermore National Laboratory, Livermore, CA 94550 USA \\ Draft version October 23, 2018
}

\begin{abstract}
Observations of type Ia supernovæ at high redshifts have become an important tool for studying the geometry of the universe. The relation between the duration of the peak phase of a type Ia supernova's lightcurve and its luminosity (broader is brighter) forms the cornerstone of this measurement, yet it is a purely empirical relation. In this paper we show that the relation is a natural consequence of the radiation transport in type Ia supernovae and suggest constraints on the nature of the explosions which arise from our interpretation of the observed relation. The principle parameter underlying the relation is the mass of radioactive ${ }^{56} \mathrm{Ni}$ produced in the explosion. The relation is shown to be relatively insensitive to most other parameters in Chandrasekhar-mass explosions. Cosmological results are thus unlikely to suffer from systematic effects stemming from evolution in the explosions' progenitors.
\end{abstract}

Subject headings: supernovae:general, cosmology:distance scale, radiative transfer

\section{INTRODUCTION}

Because they are among the brightest optical explosions in the universe, the promise of employing type Ia supernovæ (SNe Ia) as cosmological probes has long been recognized (Kowal 1968). In the past few years, this promise has been realized by two groups (Schmidt et al. 1998; Perlmutter et al. 1999) who have used observations of SNe Ia to determine luminosity distances to supernovæ at redshifts beyond $z=1$. The results have been surprising: ours is a universe which is expanding at an accelerating rate. In several ways this result is in accord with our expectations. The observations imply that the age of the universe is near $14 \mathrm{~Gy}$, comfortably older than the age of its contents. They also imply that the density of ordinary matter is well below the critical density, near $\Omega_{\text {matter }}=0.3$, and this too is in agreement with most dynamical measurements Perlmutter et al. (1999). Perhaps the most exciting result, and certainly the most unnerving and controversial, is that the geometry of the universe is today dominated by an energy density which does not arise from ordinary matter but which is best fit by a vacuum energy density for which there are no satisfying explanations (Riess et al. 1998; Garnavich et al. 1998).

To be really useful in the classic cosmological test of the redshift/luminosity-distance relation, the luminosity of a standard candle must have a small dispersion. The set of "typical" SNe Ia used for cosmology exhibits a range of almost a factor of three in peak brightness. This would not be sufficient to enable the current sample, which extends to a redshift of $z \sim 1$, to place useful limits on cosmological acceleration; the observed departure from a flat, matter-dominated universe amounts to three tenths of a magnitude ( $\sim 30 \%$ in brightness). SNe Ia are useful as cosmological probes only because what variations are observed can, for the most part, be calibrated out, leading to a usefully small dispersion in corrected brightness of about $0.15 \mathrm{mag}$.

Phillips (1993) demonstrated from a set of nearby SNe Ia that the rate of decline from peak in the optical lightcurve is correlated with peak brightness. Using the larger and more uniform data set provided by the Calán-Tololo supernova survey, Hamuy et al. (1996d) showed that this correlation exists in the B-, V-, and I-band lightcurves (though, interestingly, with different slopes than those determined by Phillips 1993 from the nearest supernovae). They found that not only the decline rate but also the overall width of the lightcurve centered on maximum light is correlated with peak luminosity. Using this same sample, Riess et al. (1995) also showed that the lightcurve shape could be used as a predictor of luminosity. Perhaps most suggestive from a physical point of view, using a sample of high-redshift supernovæ (for which very early time observations are more easily obtained), Perlmutter (1997) have shown that not only is there a correlation of the lightcurve shape with luminosity but that, near peak, the lightcurve is the same in all supernovæ up to a correlated scaling in time and luminosity, although quantitative errors for this relation have yet to be published.

While this width-luminosity relation (henceforward WLR) is very well substantiated observationally, it is an empirical relation which has remained largely unexplained. Höflich et al. (1996) showed that many current explosion models come close to this relation and suggested that the relation was determined primarily by the precise nature of the explosion itself. In this paper we provide the rudiments of a physical explanation for the WLR which is based upon the physics of radiation flow in SNe Ia, one in which the details of the explosion itself are largely masked. If true, this argues strongly against any significant bias arising in the WLR due to evolutionary effects in the supernova progenitors.

${ }^{1}$ Lawrence Livermore National Laboratory, Livermore CA 94550 USA

${ }^{2}$ Department of Astronomy and Astrophysics, University of California, Santa Cruz, Santa Cruz CA 95064 USA 
SNe Ia are widely believed to result from the thermonuclear incineration of an accreting carbon/oxygen white dwarf. Beyond this, however, little is known with any degree of precision. Present uncertainties include the nature and evolution of the progenitor system, the mass of the dwarf at ignition, and the physics of the subsequent nuclear burning. Given this state of our knowledge, it would at first blush appear hopeless to explain the WLR from physical principles. It may be, however, that the calibrating relation arises naturally from conditions present in many or all such explosions of accreting dwarfs and that the details of progenitor evolution and burning physics are of little consequence to the WLR, though fascinating in their own right.

For want of any more certain starting point, we adopt this point of view and examine how far it may take us toward an understanding of the WLR for SNe Ia. In the first section, we reprise some of the physics of radiation transport in SNe Ia explored in two previous papers (Pinto \& Eastman 2000b,a, hereafter PE I \& II). We then develop a schematic description of the lightcurve physics which illustrates the two main properties of the explosion which determine the WLR: the mass of radioactive ${ }^{56} \mathrm{Ni}$ produced in the explosion and the rate of change of $\gamma$-ray escape. In the following sections we then present a sample set of lightcurve calculations which demonstrate the essential correctness of this view, and demonstrate that the observed WLR can be reproduced with this physics. We conclude with speculations on the probability that evolutionary effects can materially alter the relation observed in the local universe and lead to false cosmological conclusions.

\section{THEORY}

The WLR is the observation that the greater the luminosity at maximum light, the greater the width of the light curve, or, equivalently, the slower the rate of decline from maximum. As discussed in PE I, the lightcurve of a SN Ia is determined by a competition between three effects: the deposition of energy from radioactive decay, the adiabatic conversion of internal energy to kinetic energy of expansion, and the escape of internal energy as the observed lightcurve.

Because the initial configuration is compact and at high density, the explosion is at first tremendously optically thick. The timescale associated with escape of internal energy is very long, and virtually all of the energy liberated in the explosion is converted to kinetic energy on a timescale approximately equal to the elapsed time. Were it not for the continuous energy input from decay of ${ }^{56} \mathrm{Ni}$ and ${ }^{56} \mathrm{Co}$ produced in the explosion, SNe Ia would be both faint and shortlived. Fortunately, ${ }^{56} \mathrm{Ni}$ decay yields an energy of $3.0 \times 10^{16} \mathrm{erg} \mathrm{g}^{-1}$ and ${ }^{56} \mathrm{Co}$ decay yields a further $6.4 \times 10^{16} \mathrm{erg} \mathrm{g}^{-1}$.

As the star expands, the optical depth decreases and the timescale over which this energy can escape declines. While trapped, radiant energy is constantly being converted into kinetic energy; the longer the radiation remains in the supernova, the less energy will escape to power the lightcurve. With a constant energy source, the luminosity would increase monotonically, asymptotically to balance the deposition at zero optical depth. In a real supernova, however, the deposition declines as radioactive material decays away and as the $\gamma$-rays, in which the decay energy is emitted, escape. Peak luminosity thus occurs roughly when the product of the rising escape fraction and the decreasing deposition reaches a maximum. This is complicated somewhat by the fact that the balance is not instantaneous; the energy emerging as UV to IR photons at any time near peak light has been deposited over a significant fraction of the elapsed time. Arnett (1982) first demonstrated that at maximum light the instantaneous bolometric luminosity is approximately equal to the instantaneous rate of energy deposition by radiative decay. Earlier, the expansion timescale is short, the escape time is long, and the deposition rate is high. More internal energy goes into expansion than escapes, and a store of trapped energy is built up. Subsequent to maximum light, more energy escapes than is converted to kinetic energy, and the surviving store of trapped energy is released faster than the deposition.

The WLR is thus seen to be a relation between the maximum-light luminosity and the radiation escape time; the brighter the supernova, the longer its escape time. Radiation escapes from an optically thick medium by diffusion, and we can tentatively identify the escape time with the diffusion time - the average time it takes a photon to random walk its way to the surface, given by the number of scatterings times the time of flight between scatterings.

Following the usual random walk argument, the diffusion time can be written as

$$
t_{d}=\frac{\alpha R \tau_{\text {eff }}}{c}
$$

where $R$ is a characteristic size of the system (in this case, the radius of the ejecta), $\tau_{\text {eff }}$ is the effective optical depth, and $\alpha$ is a constant of order unity. By the argument above, if the expansion (elapsed) time and escape (diffusion) times are about equal at peak, we can write

$$
t_{d}=t=\frac{\alpha R \tau_{e f f}}{c}=\frac{\alpha V t \tau_{e f f}}{c}
$$

which gives a diffusion optical depth at peak of

$$
\tau_{\text {eff }} \approx 30\left(\frac{10^{9} \mathrm{~cm} \mathrm{~s}^{-1}}{V}\right)
$$

(taking $\alpha \approx 1$ ). This value is far smaller than the monochromatic optical depth to most trapped photons, which is typically nearer to 3000 for peak conditions in a SN Ia. The resolution to this seeming paradox, of course, is that the typical photons in an atmosphere do not carry the flux - radiation escapes where it can, at energies where the monochromatic optical depth is smaller.

At stellar densities, there is tight collisional coupling between the radiation field and the plasma, keeping the local photon distribution function at its blackbody limit. While the bulk of the energy density exists as UV photons, these 
photons are trapped by the high UV opacity. The bulk of the flux is transported at longer wavelengths on the RayleighJeans tail of the distribution, where the optical depth is typically less, "side-stepping" the higher UV opacity. The lower density of these photons is more than overcome by the decreased diffusion time they suffer, and the appropriate opacity to use in describing the flux is the familiar Rosseland mean.

For this picture to work, the relaxation rate of the trapped photon distribution toward a blackbody must be short compared with the rate at which energy escapes from the long-wavelength tail of the distribution. This relaxation is usually mediated by collisions, and its rate must therefore scale as the square of the density. If the optical depth to the majority of photons remains large (so that radiation remains trapped) while the density decreases, eventually collisions will not be able to maintain sufficient energy density in the long-wavelength tail of the photon distribution where the optical depth is less; the system will depart from local thermodynamic equilibrium (LTE).

PE II demonstrated that this has indeed become the case in SNe Ia by maximum light. The escape time is still mediated by the rate for converting energy from short-wavelength, UV photons into long wavelength, OIR photons. The difference from the more typical LTE situation arises from the nature of opacity in rapidly-expanding media and the character of atomic physics in iron-group ions.

In the homologous expansion which develops soon after explosion, the velocity gradient is isotropic. Much as in the expanding universe, there is a one-on-one mapping between path-length and redshift, and a photon traversing the ejecta will accumulate a red shift in the local gas rest frame which will bring it into resonance with line transitions. One can show (Eastman \& Pinto 1993) that under the conditions in SNe Ia, the time a photon spends scattering in a line is very short compared with the time it spends moving between lines. For the purpose of estimating the escape time, then, each line traversed thus acts as a single scattering, and the distance measured in units of a photon mean free path (the optical depth) will be just the number of optically thick lines traversed in accumulating a given Doppler shift; the corresponding effective opacity is known as an "expansion opacity" ( $c f$. Eastman \& Pinto 1993; Wagoner et al. 1991; Karp et al. 1977; Castor et al. 1975).

Using simple analytic estimates, PE I showed that the central temperature in a $\mathrm{M}_{\text {ch }}$ explosion at $t<20$ days is $T \gtrsim 13,000 \mathrm{~K}$ and the peak of the blackbody spectrum is in the UV near $\lambda \lesssim 2200 \AA$. For an iron-peak composition in the relevant range of temperature and density, the spectral density of optically thick lines in the UV is very large and is a strongly decreasing function of wavelength from the UV through the optical (cf. Figure 2 of PE II). As long as the Sobolev optical depth in these lines remains large, the diffusion optical depth will not change as the density decreases, allowing a departure from LTE not seen at depth in static atmospheres where the optical depth is linearly dependent upon density. The large spectral density of lines at UV wavelengths is far too great for there to be significant transport at UV energies; the time it takes a UV photon to random walk to the surface is long compared to the expansion time. This is substantiated by the observation of very little UV flux in maximum light SNe Ia in spite of their high temperatures.

If the Rayleigh-Jeans tail of the photon distribution (at wavelengths where significant transport can take place) could be re-populated by a mechanism more efficient at low densities than collisional excitation, significant flux could still be developed, leading to an escape time characterized by the required effective optical depth near 30 .

When a photon is absorbed in a line, the possible outcomes of this interaction include: 1) re-emission in the same transition, followed possibly by re-absorptions and emissions - a cycle repeated many times until escape from the red wing of the line; 2) collisional destruction, transferring the photon energy by Coulomb interactions to a free electron; and 3) fluorescence, where the excited state populated by absorption de-populates by radiative decay to a state different from the original. In general, the electron density is too low in maximum light SNe Ia for collisional destruction to have much effect. Thus, a UV photon absorbed on the high energy (blue) side of a line will either be re-emitted on the red side, or fluorescence will occur, with the photon replaced by several longer-wavelength photons (usually), but occasionally by a shorter wavelength photon (less often). The efficiency for this conversion process obviously depends on the number and strength of decay channels which would result in the emission of an OIR photon. For the UV radiation trapped in SNe Ia, fluorescence is the most likely outcome. Fluorescence is much more important in SNe Ia than in other astrophysical objects because they are composed in large part of iron-group elements with enormously complex atomic structures and very high spectral densities of line transitions in which fluorescence can occur.

A key point is this: the number of OIR transitions is greatest in the neutral and low ionization states, and decreases with increasing ionization. Thus the UV $\rightarrow$ OIR fluorescence process is more efficient in Co II than in Co III, and more efficient in Co III than in Co IV. Consequently, the conversion process is sensitive to the ionization. In the approximate calculations reported below, we assume LTE ionization and excitation despite densities which are too low for this to be really true. We shall thus use temperature as a proxy for ionization, but the reader should keep in mind that this is an approximation, and it is actually ionization which is the controlling variable.

In light of the importance of fluorescence, we can sketch the escape of radiation from SNe Ia in the following fashion. Imagine a store of deposited energy, trapped in the form of UV photons. These UV photons are created by the absorption and thermalization of decay $\gamma$-rays and removed by conversion to kinetic energy and to OIR photons. For the energy density of UV photons, $E_{U V}$, we can write the following schematic rate equation:

$$
\frac{\partial E_{U V}}{\partial t}=\rho \dot{S}_{d e p}(t)-\frac{E_{U V}}{t_{e}}-r_{f} E_{U V}
$$

where $\rho \dot{S}_{d e p}(t)$ is the time-dependent deposition rate, $E_{U V} / t_{e}$ is the rate of conversion of radiation to kinetic energy, and $r_{f} E_{U V}$ is the rate for converting UV to optical and infrared energy. We have assumed here that the diffusion time suffered 
by the UV energy is infinitely long; transport in the UV is unimportant. Similarly, for the OIR photons one can write

$$
\frac{\partial E_{O I R}}{\partial t}=-\frac{E_{O I R}}{t_{e}}-\frac{E_{O I R}}{t_{d}}+r_{f} E_{U V}
$$

where $t_{d}$ is the typical OIR diffusion time. Here we have ignored the possibility that deposition results in the direct emission of OIR photons. If optical photons are processed on a short enough time scale - for instance, if $t_{d} \lesssim t_{e}-$ then we can set $\partial E_{O I R} / \partial t \approx 0$ and solve for $E_{O I R}$ :

$$
E_{O I R}=\frac{r_{f}}{1 / t_{e}+1 / t_{d}+r_{f}} E_{U V} .
$$

Finally, by adding equations (4) and (5) together, and making use of equation (6), we obtain an equation for the total energy density, $E_{t o t}$ :

$$
\frac{\partial E_{t o t}}{\partial t}=\rho \dot{S}_{d e p}(t)-\frac{E_{t o t}}{t_{e}}-\frac{E_{t o t}}{\tilde{t}_{d}}
$$

where the effective diffusion time, $\tilde{t}_{d}$, is defined as

$$
\tilde{t}_{d} \equiv \frac{t_{d}+t_{e}+r_{f} t_{d} t_{e}}{r_{f} t_{e}} .
$$

Written this way, it is easy to see that as $r_{f}$ - the UV to OIR fluorescence rate - decreases, the effective diffusion time goes up. In terms of the opacity, $r_{f}$ can be written in LTE as

$$
r_{f}=c \rho\left(\frac{\int_{\nu_{O I R}}^{\infty} \kappa_{\nu^{\prime}} B_{\nu^{\prime}} d \nu^{\prime}}{\int_{\nu_{O I R}}^{\infty} B_{\nu^{\prime}} d \nu^{\prime}}\right) \times\left(\frac{\int_{0}^{\nu_{O I R}} \kappa_{\nu^{\prime}} B_{\nu^{\prime}} d \nu^{\prime}}{\int_{0}^{\infty} \kappa_{\nu^{\prime}} B_{\nu^{\prime}} d \nu^{\prime}}\right)
$$

where $B_{\nu}$ is the Planck function at the local gas temperature, and $\nu_{O I R}$ is a frequency which approximately divides the UV from the optical. The first fraction is just the Planck mean opacity in the UV, and the second fraction is the fraction of absorbed energy which is radiated at optical wavelengths. Since, for instance, Co II has a larger opacity in the optical than Co III, increasing the ionization from once- to twice-ionized will decrease the fraction of absorbed energy which is re-radiated in the optical, making $r_{f}$ smaller, and therefore increasing the effective diffusion time.

We can now understand the underlying physics of the WLR: as the amount of ${ }^{56} \mathrm{Ni}$ is increased, the interior temperature and ionization go up, which decreases $r_{f}$ and increases $t_{d}$. Stated another way, brighter SNe Ia (i.e. ones with more ${ }^{56} \mathrm{Ni}$ ) are hotter inside, and therefore have longer diffusion times (i.e. evolve more slowly through peak).

This cannot be the whole story, however. As long as most of the ${ }^{56} \mathrm{Ni}$ is unmixed and its mass fraction $X_{56} \approx 1$ in the ${ }^{56} \mathrm{Ni}$-dominated layers, increasing the total amount of ${ }^{56} \mathrm{Ni}$ in the star does not increase the local heating rate per gram, and therefore would not, by itself, result in a temperature increase. Overlooked so far is the fact that, if the additional ${ }^{56} \mathrm{Ni}$ is added at higher velocity, then there will be an increase in the effective optical depth. That is, in stars with more ${ }^{56} \mathrm{Ni}$ (in a layered composition), the velocity of the outer edge of the ${ }^{56} \mathrm{Ni}$ is moving faster than is the outer edge of the ${ }^{56} \mathrm{Ni}$ in a star with less ${ }^{56} \mathrm{Ni}$. The higher the velocity of the outer edge of the ${ }^{56} \mathrm{Ni}$, the more lines through which a photon must Doppler shift before escaping. This represents an optical depth increase which would increase the escape time and therefore make the gas hotter. Putting iron-peak elements at higher velocity is, by itself, not sufficient to increase $\tau_{e f f}$ enough to fully explain the observed correlation between light curve width and luminosity. Rather, the slight increase in optical depth provided by putting ${ }^{56} \mathrm{Ni}$ at higher velocities is amplified by its effect on temperature and ionization, and thus the escape time. It is difficult to cleanly separate these two effects, but in the next section we present results which strongly suggest that ionization is the dominant mechanism determining the escape time.

It is worth stressing here that fluorescence is not a one-way process. Energy can be transported to shorter wavelengths by "combining" photons. A photon can be absorbed, raising an ion to an excited state. The ion can then absorb another photon into this state, raising it to yet a higher-energy state from which a UV photon can be emitted. From obvious thermodynamic considerations (embodied in Rosseland's Theorem of Cycles) this "combining" of photons must in general be less frequent than "splitting" of UV photons. Near the peak in the lightcurve, however, over much of the volume of the supernova these processes are nearly in equilibrium; it is the net rate of transport of energy to longer wavelengths which determines the escape time, not merely a one-way fluorescent cascade. Indeed, Mazzali (2000) has suggested that "combining", in lower-optical depth regions above the photosphere, is a significant contributor to the UV emission at maximum light.

Another avenue for time-dependence to enter the equations above is through the energy deposition term $\dot{S}_{d e p}(t)$. The deposition is determined both by how much ${ }^{56} \mathrm{Ni}$ is present and by what fraction of its decay energy is deposited in the gas. Because the Compton opacity to $\mathrm{MeV} \gamma$-rays is mainly absorptive, the deposition fraction is roughly proportional to $1-\exp \left(-\tau_{\gamma}\right)$, where $\tau_{\gamma}$ is the mean gamma-ray optical depth and is in turn roughly proportional to the mean column depth to ${ }^{56} \mathrm{Ni}$. More-rapid expansion will lead to a lower mean optical depth to $\gamma$-rays at a given time, as will mixing radioactive material to higher velocities. Lower column depths will in turn lead to a more-rapid decline in the deposition fraction. If $\dot{S}_{d e p}(t)$ declines more rapidly, the lightcurve will peak earlier. An earlier peak will have more ${ }^{56} \mathrm{Ni}$ still present, and this will tend to offset the larger escape fraction. The lightcurve will thus evolve more rapidly, leading to a narrower lightcurve for it luminosity.

We have thus identified three properties of the explosion which may have a strong effect upon the WLR. The mass of ${ }^{56} \mathrm{Ni}$ determines the ionization and through it the mean escape time. The expansion velocity (or specific kinetic energy) 
determines the rate of decline of the energy deposition. The radial distribution of ${ }^{56} \mathrm{Ni}$ can have an effect upon both the escape time of the thermalized radiation and the time evolution of the deposition. In the following sections we present a small set of synthetic lightcurves which illustrate these effects and their relative importance.

\section{COMPUTATIONS}

A complete model for supernova lightcurves would begin with a model for the evolution of the progenitor. This would be evolved to ignition, the subsequent explosive burning followed to completion, and a transport calculation performed to determine the lightcurve. Unfortunately, a fully predictive model is not yet available from such a procedure. While there are schematic models for all of these stages, it is not yet possible to simulate SN Ia explosions without introducing very simple parameterizations to stand in for very complex physics we do not yet understand.

The evolution of the progenitor is undoubtedly the most uncertain part of this process, yet there is the possibility that "convergent evolution" of some sort, perhaps ignition near the Chandrasekhar mass in single degenerate systems, reduces the sensitivity to initial conditions. On the other hand, the initial conditions in the various double-degenerate scenarios might well be the single most important piece of physics determining the subsequent explosion. In the burning phase, the physics of turbulent combustion and the possible spontaneous transition to detonation are probably the most important and least tractable effects. While progress is being made on all of these fronts (Reinecke et al. 1999; Iwamoto et al. 1999), it will be a long time before truly predictive models of progenitor evolution and explosion become available.

Our aim in this work is to explore the radiation transport effects which influence the WLR. We have thus chosen to construct a very simple model for the structure of the explosion. We start with model DD4 of Woosley \& Weaver (1991), an $\mathrm{M}_{\mathrm{ch}}$ explosion which burned $1.26 \mathrm{M}_{\odot}$ to $\mathrm{Si}$ or heavier elements to yield $1.2 \times 10^{51}$ ergs of kinetic energy, producing 0.63 $\mathrm{M}_{\odot}$ of ${ }^{56} \mathrm{Ni}$. We have modified the mass and distribution of ${ }^{56} \mathrm{Ni}$ in this model to explore the effects such modifications have on the lightcurve. The density as a function of velocity in the original model DD4 is preserved in all models. The structure of DD4 is presented by Woosley \& Weaver (1991) and by PE II.

An initial grid of models with different ${ }^{56} \mathrm{Ni}$ masses was obtained by mapping between ${ }^{56} \mathrm{Ni}$ and ${ }^{28} \mathrm{Si}$ according to

$$
\begin{aligned}
& X_{56}(M)=X_{56}^{0}(M) \exp (-\phi(M)) \\
& X_{28}(M)=X_{28}^{0}(M)+X_{56}^{0}(M),(1-\exp (-\phi(M)))
\end{aligned}
$$

where

$$
\phi(M) \equiv \max \left(\alpha\left(M-M_{c u t}\right), 0\right) .
$$

To increase the ${ }^{56} \mathrm{Ni}$ mass, all isotopes below $M=M_{\text {cut }}$ were mapped to ${ }^{56} \mathrm{Ni}$ :

$$
X_{56}(M)=1-H\left(M-M_{c u t}\right)+X_{56}^{0}(M) H\left(M-M_{c u t}\right),
$$

where $H(x)$ is the Heaviside function, and for $A \neq 56$,

$$
X_{A}(M)=X_{A}^{0}(M) H\left(M-M_{c u t}\right) .
$$

This simple scheme is actually well-motivated in a physical sense. The total energy released from burning a $\mathrm{C} / \mathrm{O}$ mixture to intermediate elements in the Si group is very nearly the same as for burning to iron-peak elements. The ${ }^{56} \mathrm{Ni}$ mass thus need have little necessary connection to the explosion kinetic energy. The most stringent constraints on the distribution of elements in velocity come from peak-phase spectroscopy, though this analysis is hampered by our inability to compute time-dependent NLTE models.

As discussed in PE I, the initial thermal energy in the explosion goes entirely into kinetic energy via the second term on the r.h.s. of equation (4) and hence has no effect upon the observable part of the lightcurve. All the models presented here were started with the same initial internal energy.

The lightcurves were computed as described in PE II, using time-dependent, multi-group radiation transport with an LTE equation of state and including the elements He, C, O, Si, S, Ca, Fe, Co, and Ni in ionizations up to Ni XIII. 6000 energy groups were employed, providing moderate resolution; doubling the number of groups does not significantly alter the results, though halving it does leads to marked effects. We are forced to employ LTE level populations because of computer-time constraints - a NLTE calculation would take as long per time step as a whole lightcurve in LTE (several days on a fast workstation). All current NLTE calculations (Höflich 1995; Nugent et al. 1997; Mazzali \& Lucy 1993) employ temperature structures which are not consistent with the history of the ejecta's evolution and therefore cannot be expected to give correct time-dependent spectra and lightcurves, as discussed in PE II. While these LTE calculations obviously suffer as well, the ability to include time-dependent physics correctly is evidently necessary to study the effects discussed above.

The WLR is an observed relation between peak magnitude and a measure of width. To obtain an accurate result in the face of measurement error and the temporal sparsity of observed data, both the peak magnitude and the width are measured by fitting observed data to templates determined from well-observed supernovae (Hamuy et al. 1996b; Riess et al. 1995). It is important to employ the same methods to measure the peak magnitude and width in our models. We "observe" our synthetic lightcurves by employing the MCLS (Multi-Color Lightcurve Shape) method of Riess et al. (1995), employing the revised vectors used in Riess et al. (1998) (kindly provided by Dr. Riess). We have also determined the $\Delta m_{15}(B)$ decline-rate parameter for our models using the $\chi^{2}$ template-fitting technique described by Hamuy et al. (1996c). The results of fitting from both of these techniques are shown in table 1.

We note that the observational difficulty of obtaining early lightcurve data has hindered the construction of templates for times earlier than ten days before peak. Thus, by construction, template-fitting techniques are most sensitive to 
post-maximum decline rates; their ability to discriminate between different pre-maximum lightcurve shapes is seriously hampered.

Observationally, WLR relations with similar scatter exist in the B, V, R, and I bands - color evolution during the first 30 days is well correlated with luminosity and width. At later times, all SNe Ia show a remarkable homogeneity in color. In the absence of reddening, determining the width of the lightcurve in a single band is sufficient to uniquely determine a supernova's peak magnitude. The importance of fitting a supernova's color evolution is primarily in determining extinction. As our models do not suffer from this observational uncertainty, it is sufficient to fit to a single band.

We remind the reader as well that individual supernovae show significant departures from the best-fit templates $(c f$. Hamuy et al. 1996a); SNe Ia do not form a strictly one-parameter family. While the width of lightcurves measured by the templates is the largest variation from object to object, there are other features in the lightcurve which are not correlated with the decline rate. A good example of this is the pair SN1991T and SN1992bc which have very similar decline rates over the first 15 days past peak but very different rates over the subsequent 45 days (Hamuy et al. 1996c).

The details of the atomic physics and NLTE effects determine the color evolution of the supernova. We have assumed in this study that such effects are less important to the bolometric luminosity, merely reapportioning flux between the B, $\mathrm{V}$, and $\mathrm{R}$ bands which comprise most of the luminosity (Suntzeff 1995). We have thus chosen to take fits to the WLR in the $\mathrm{V}$ band only as most representative of the overall relation between width and luminosity. Below we present fits to $\mathrm{B}, \mathrm{V}$, and R simultaneously as well, but as an accurate determination of the color evolution depends more upon specific details of the atomic models and NLTE effect, we regard these fits as less reliable indicators of the underlying physics than the trends in a single band.

As the ejecta evolve, the effects of trapping and fluorescence become less important to the bolometric lightcurve. The colors at these later times, however, are very strongly influenced by NLTE effects. LTE calculations cannot be expected to provide an adequate representation of the lightcurve physics as the supernova becomes a nebula. Thus, we fit only to the peak phase of the lightcurve and estimate the sensitivity of fitting to various time intervals by fitting from 10 days before peak (the earliest time in the templates) until 20 and 40 days past peak.

\section{RESULTS}

Figure (1) compares the MCLS $\Delta$ parameter (a measure of width) to the peak V absolute magnitude determined from the models described above. The MCLS method parameterizes the shape of a supernova's lightcurve as

$$
M(t)=A(t)+\Delta \times L(t)+\Delta^{2} \times Q(t)
$$

where $A(t)$ is, roughly, the mean lightcurve shape of a sample of 27 well-observed supernovae (Riess et al. 1998) and $L(t)$ and $Q(t)$ are functions describing a quadratic fit to the departure of individual supernovae from this mean shape as

TABLE 1

MCLS LightCURVE Fits

\begin{tabular}{|c|c|c|c|c|c|c|c|c|c|c|}
\hline model & $\begin{array}{c}\mathrm{M}\left({ }^{56} \mathrm{Ni}\right) \\
\left(\mathrm{M}_{\odot}\right)\end{array}$ & $\begin{array}{c}M_{V}^{\text {peak }} \\
\text { (actual) }\end{array}$ & $\Delta m_{15}{ }^{\mathrm{a}}$ & $\begin{array}{c}M_{V}^{\text {peak } \mathrm{b}} \\
\left(\Delta m_{15}\right)\end{array}$ & $\Delta_{20}{ }^{c}$ & $\begin{array}{l}M_{V}^{\text {peak } \mathrm{d}} \\
\left(\Delta_{20}\right)\end{array}$ & $\Delta_{40}{ }^{\mathrm{e}}$ & $\begin{array}{l}M_{V}^{\text {peakf }} \\
\left(\Delta_{40}\right)\end{array}$ & $\begin{array}{c}\Delta_{20}{ }^{\mathrm{g}} \\
(\mathrm{BVR})\end{array}$ & $\begin{array}{c}\Delta_{40}{ }^{\mathrm{h}} \\
(\mathrm{BVR})\end{array}$ \\
\hline $\mathrm{DD} 4 / 27$ & 0.27 & -18.90 & 1.42 & -18.87 & 0.470 & -18.98 & 0.310 & -18.87 & 0.509 & 0.479 \\
\hline DD4/38 & 0.38 & -19.17 & 1.47 & -19.15 & 0.348 & -19.10 & 0.241 & -19.21 & 0.460 & 0.427 \\
\hline DD4/48 & 0.48 & -19.34 & 1.37 & -19.34 & 0.121 & -19.33 & 0.159 & -19.23 & 0.220 & 0.235 \\
\hline DD4/55 & 0.55 & -19.42 & 1.24 & -19.43 & -0.054 & -19.51 & 0.123 & -19.47 & 0.160 & 0.157 \\
\hline DD4 & 0.64 & -19.52 & 1.22 & -19.52 & -0.123 & -19.58 & 0.131 & -19.32 & 0.160 & 0.157 \\
\hline DD4/90 & 0.90 & -19.78 & 0.87 & -19.81 & -0.443 & -19.91 & -0.542 & -20.02 & -0.704 & -0.710 \\
\hline M1 & 0.64 & -19.56 & 1.24 & -19.55 & -0.126 & $\begin{array}{c}-19.58 \\
\end{array}$ & 0.018 & $\begin{array}{c}-19.43 \\
\end{array}$ & 0.219 & 0.244 \\
\hline $\mathrm{NiFe}$ & 0.32 & -19.01 & 1.75 & -19.01 & 0.633 & -18.81 & 0.502 & -18.94 & 0.655 & 0.543 \\
\hline DD3 & 0.93 & -19.90 & 0.87 & -19.90 & 0.083 & -19.36 & -0.161 & -19.62 & -0.698 & -0.525 \\
\hline
\end{tabular}

${ }^{\text {a }} \Delta m_{15}$ measured from the $\mathrm{V}$ lightcurve.

${ }^{\mathrm{b}}$ Peak $\mathrm{V}$ magnitude as measured by the $\Delta m_{15}$ fit.

${ }^{\mathrm{c}}$ MCLS width measured from the V lightcurve from $(-10,20)$ days.

${ }^{\mathrm{d}}$ Peak V magnitude as measured by the $\Delta_{20}$ fit.

e MCLS width measured from the V lightcurve from $(-10,40)$ days.

${ }^{\mathrm{f}}$ Peak $\mathrm{V}$ magnitude as measured by the $\Delta_{40}$ fit.

${ }^{\mathrm{g}}$ MCLS width measured from the B, V, and R lightcurves from $(-10,20)$ days.

${ }^{\mathrm{h}}$ MCLS width measured from the B, V, and R lightcurves from $(-10,40)$ days. 


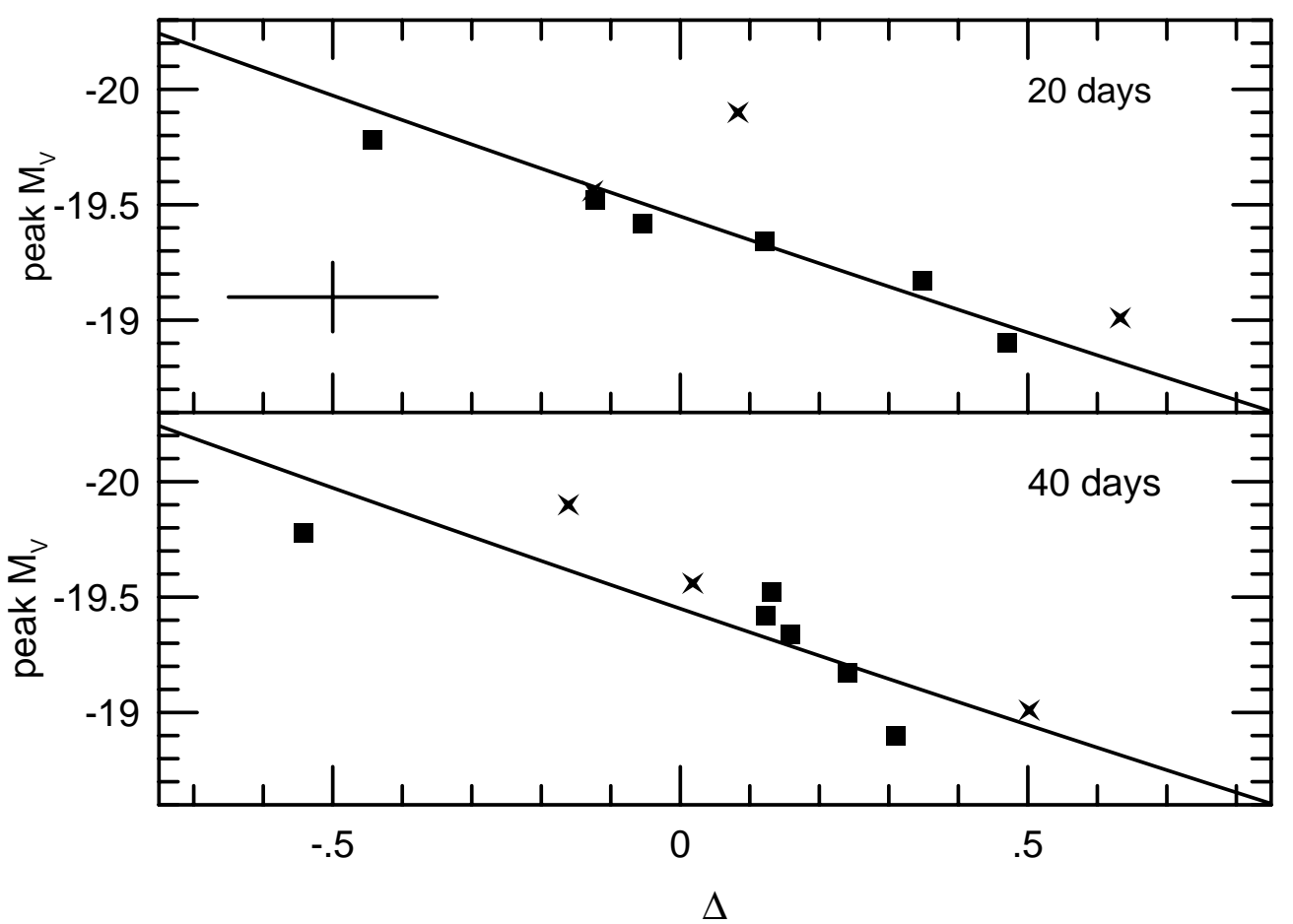

FIG. 1.- Peak V magnitudes vs the MCLS $\Delta$ parameter (a measure of lightcurve width) fit to V lightcurves over the interval (-10,20) days (top panel) and $(-10,40)$ days (bottom panel) from peak. The squares correspond to calculations made by varying the ${ }^{56} \mathrm{Ni}$ mass in model DD4. The stars show the three variations from these models as discussed in the text. The diagonal curve in each panel is the observed quadratic relation between $M_{V}^{\text {peak }}$ and $\Delta$ using the Cepheid calibration of SNe Ia. The approximate magnitude of the observed dispersion about this relation, corresponding to $\pm 0.15 \mathrm{mag}$ and \pm 0.125 in $\Delta$, is indicated in the top panel

parameterized by $\Delta$ which is thus a measure of lightcurve width. The quadratic relation between $\Delta$ and $V_{\text {peak }}$ is indicated by the diagonal curves in the figure. The absolute luminosity of the relation is calibrated by the Cepheid distance scale of (Gibson et al. 2000) corresponding to a Hubble constant of $68 \mathrm{~km} \mathrm{~s}^{-1} \mathrm{Mpc}^{-1}$. The square symbols correspond to the models with $0.27,0.38,0.48,0.55,0.63$, and $0.90 \mathrm{M}_{\odot}$ of ${ }^{56} \mathrm{Ni}$ in the structure of model DD4. The star symbols represent other models to be discussed below. The top panel shows fits over the interval $(-10,20)$ days relative to peak and the lower panel over the interval $(-10,40)$. The quality of the fit to the mean WLR relation is somewhat better for the scaled- ${ }^{56} \mathrm{Ni}$ models for the shorter interval and is probably a consequence of the increasingly inappropriate assumption of LTE at later times.

In both cases, the fits show a dispersion comparable to or smaller than the observations $(\sim 0.15 \mathrm{mag})$ and (remarkably and probably fortuitously) virtually zero departure from the absolute calibration. It is clear from this figure that the $\mathrm{V}$ WLR is fit remarkably well by the simple prescription of changing only the ${ }^{56} \mathrm{Ni}$ mass.

Figure (2) shows the same lightcurves fit by the template-fitting $\left(\Delta m_{15}(B)\right)$ method of Hamuy et al. (1996b) on the interval $(-5,26)$ days. In this method, the models are fit to each of six template lightcurves spanning the range of wellobserved supernovae. The templates are characterized by their $\Delta m_{15}(B)$ parameters (roughly the decline in B magnitude over 15 days from peak), while the actual epochs fit are determined by the whole peak phase of the lightcurves. The bestfit $\Delta m_{15}(B)$ is then determined from the minimum of a quadratic fit to the reduced $\chi^{2}$ as a function of the parameter. Rather than extrapolating, models broader than the broadest template are assigned the minimum value of $\Delta m_{15}(B)$, accounting for the slight pile-up of points at the bright end of the relation.

The filled symbols are the same as those of the previous figure, and the open symbols are fits to 59 well-observed supernovae from Phillips et al. (1999). The curve is the mean $\Delta m_{15}(B)-M_{V}$ relation, on the same calibration, from (Phillips et al. 1999). Once again, the models fit the relation well within the scatter of observed supernovae. The significant difference in curvature between the mean $\Delta m_{15}(B)$-luminosity and $\Delta$-luminosity relations is notable and appears to be the result of the differing ways in which the lightcurve shapes are parameterized; both techniques achieve similar reductions in the scatter about the Hubble relation.

Figure (3) shows the V lightcurve family from the six scaled DD4 models compared with the best-fitting MCLS templates. The shapes of the lightcurves depart less than 0.05 magnitude from the templates throughout the first 45 days 


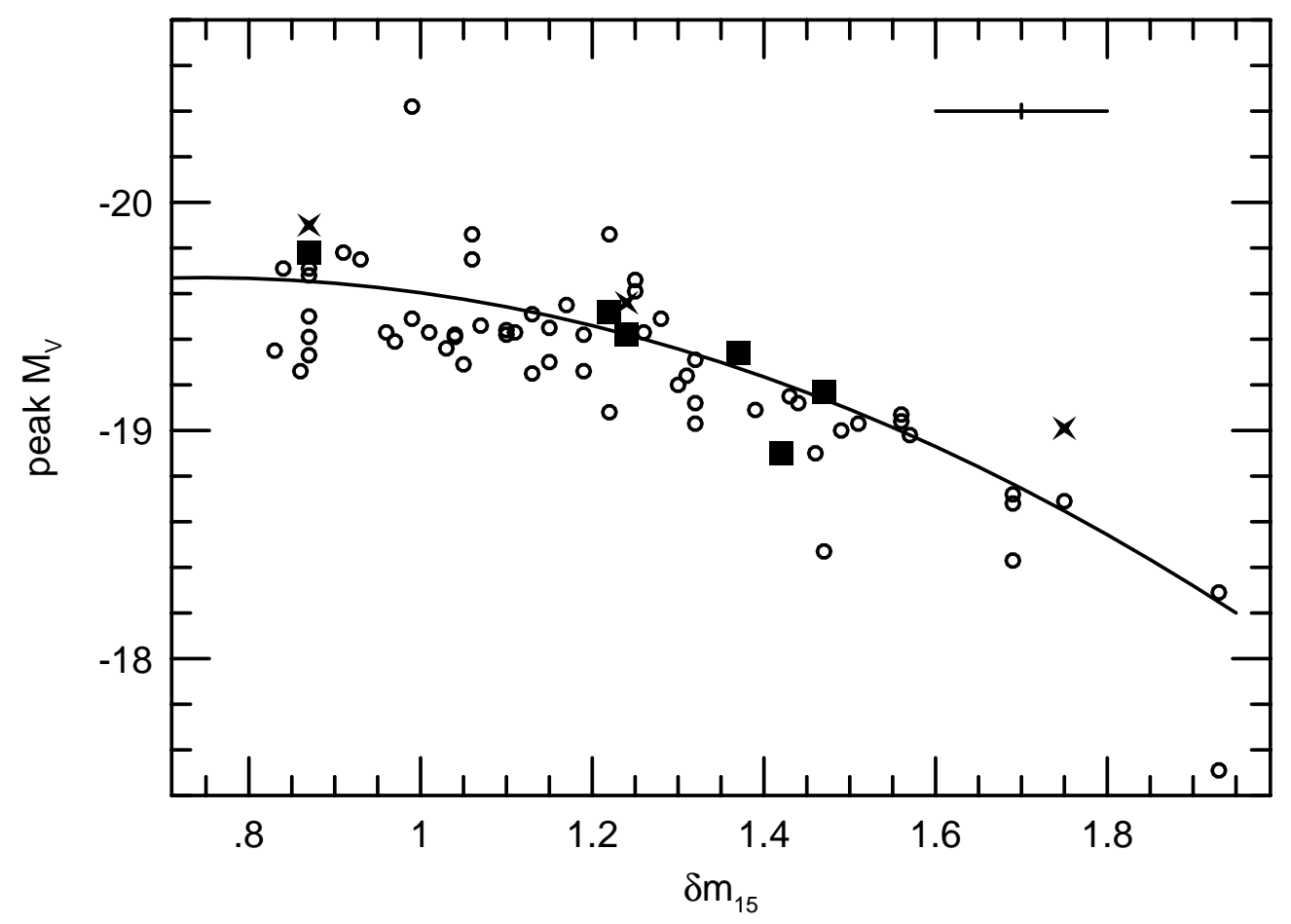

FIG. 2.- Peak V magnitudes vs the $\Delta m_{15}(B)$ parameter (another measure of lightcurve width) measured from V lightcurves and on the same luminosity calibration as the previous figure. Open symbols are data for 59 supernovae from Phillips et al. (1999), on the same calibration as in the previous figure. The stars show the three variations from the scaled-DD4 models as discussed in the text.

(about 25 days past $\mathrm{V}$ peak) with the exception of the $0.90 \mathrm{M}_{\odot}$ model, where the inflection at 32 days is not seen in the templates. This error is well within the dispersion of data from individual supernovae about the template lightcurves $(c f$. Riess et al. 1998). At later times, the LTE approximation becomes increasingly poor and may account for the too-shallow decline rates, though real supernovae show similar departures from the templates at this time.

Figures (4) and (5) show the $\mathrm{B}, \mathrm{V}$, and R lightcurves of the $0.55 \mathrm{M}_{\odot}$ and $0.90 \mathrm{M}_{\odot}$ models, respectively. The DD4/55 model displays a generic problem with most of the models; while the B lightcurve is the correct width, near peak it is too flat compared to the templates. The departure from the templates before peak is less disturbing, and is probably more a problem with the templates than the models, as there is comparatively little data so far before maximum. The IR secondary maximum is clearly seen in the R-band curves.

We now turn to variations on these scaled-DD4 models to investigate the sensitivity of the WLR to other changes in the explosion. The models presented so far have nearly pure ${ }^{56} \mathrm{Ni}$ from the center out to a mass coordinate nearly equal to the Ni mass - radioactive material is concentrated as much as possible toward the center. This is a natural result of the explosion physics. White dwarfs are densest at the center. When matter burns explosively, the higher the density, the larger the ratio of the hydrodynamic timescale to the the burning timescale, and the more the distribution of burning products is skewed toward heavier elements. In the absence of significant hydrodynamic mixing after material has cooled below the burning temperature, a natural consequence of explosive burning is that the gradient of atomic weight will point inward, with the heaviest burning product, ${ }^{56} \mathrm{Ni}$, concentrated toward the core.

To explore the effect hydrodynamic mixing might have on the lightcurve, we have altered the distribution of ${ }^{56} \mathrm{Ni}$ in model DD4 without changing its mass. A significant mass fraction of ${ }^{56} \mathrm{Ni}$ extends approximately to $0.85 \mathrm{M}_{\odot}$ in the original model (primarily as a consequence of producing $0.16 \mathrm{M}_{\odot}$ of non-radioactive iron-group material). The starred points at $M_{V}=19.56$ in Figures (1) and (2) show the result of homogenizing the composition of this model over the mass range 0.7 to $1.1 \mathrm{M}_{\odot}$ in model M1. This decreases the peak bolometric magnitude by 0.1 mag and leads to significantly narrower $\mathrm{B}$ and brighter I lightcurves. The resulting $\mathrm{V}$ and $\mathrm{R}$ magnitudes are virtually identical, 0.04 and 0.02 mag brighter, respectively. The ${ }^{56} \mathrm{Ni}$ nearer the surface cooled more rapidly. The lower ionization gas converts energy from shorter- to longer-wavelength photons more efficiently, circumventing the effect of the higher expansion optical depth from ${ }^{56} \mathrm{Ni}$ at higher velocities. Altering the distribution of ${ }^{56} \mathrm{Ni}$ would appear to affect the color of the emergent flux more than its overall escape properties. An important conclusion is that, within the limits of fitting $0.63 \mathrm{M}_{\odot}$ of ${ }^{56} \mathrm{Ni}$ in an $\mathrm{M}_{\mathrm{ch}}$ white dwarf, the WLR is insensitive to the distribution of radioactivity in the explosion. 


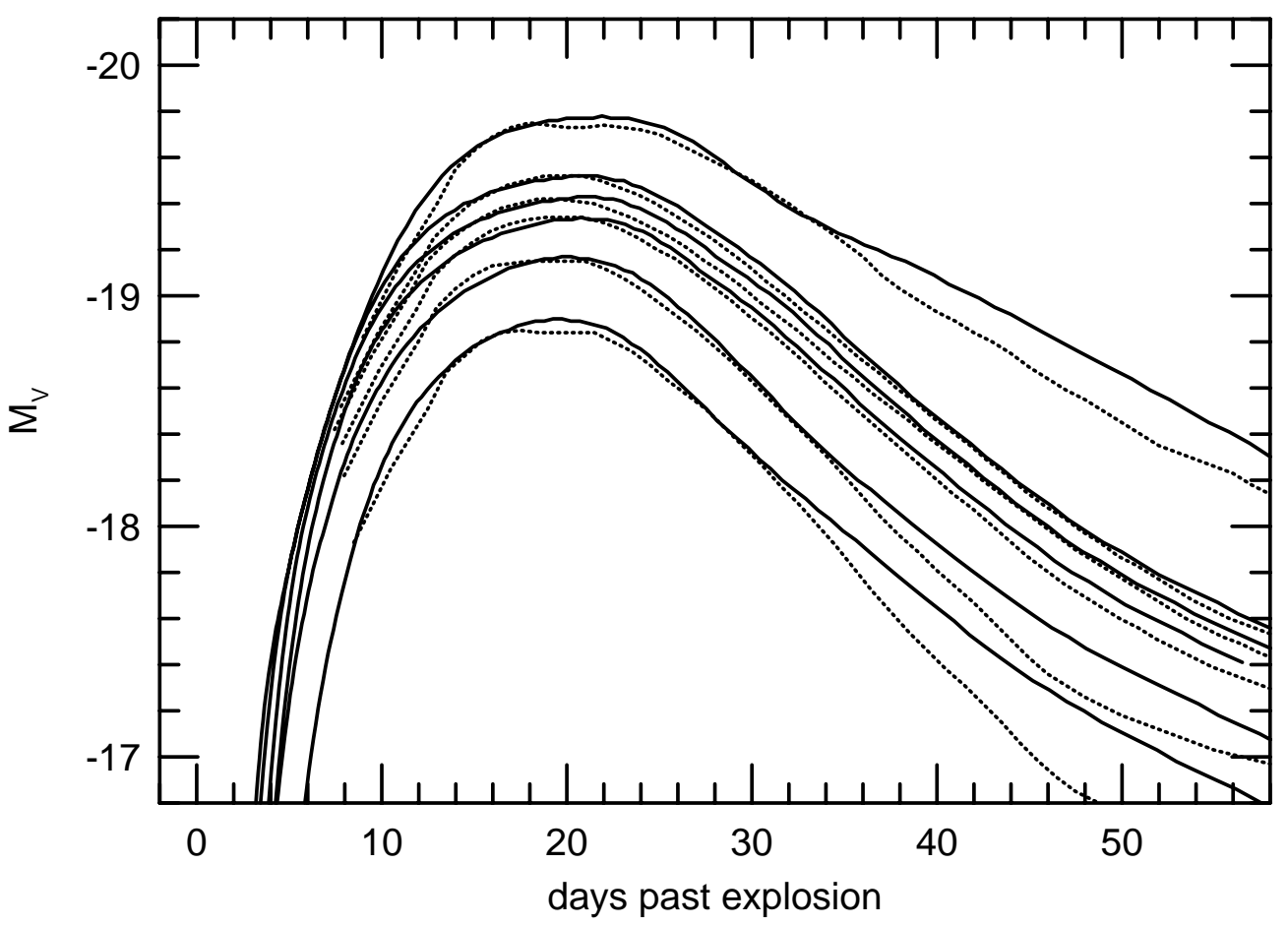

FIG. 3.- The six V scaled-DD4 lightcurves. The solid lines are the synthetic lightcurves; the dotted lines are the best-fit MCLS template lightcurves fit over the interval $(-11,20)$ days with respect to peak.

The scaled-DD4 models traded off production of ${ }^{56} \mathrm{Ni}$ with intermediate mass elements. This is the only possibility afforded if the supernovae are to satisfy nucleosynthetic constraints. For example, the production of large quantities of non-radioactive iron-group elements in the majority of supernovæ is firmly ruled out. Nonetheless, in model NiFe we have converted, in place, half of the mass of ${ }^{56} \mathrm{Ni}$ to ${ }^{56} \mathrm{Fe}$, thus changing the heating rate per gram of the iron-rich material without changing its distribution, cooling, or $\gamma$-ray escape properties. The lower temperature in the ${ }^{56} \mathrm{Ni}$-rich material leads to lower ionization and hence to more efficient escape. In spite of having 0.32 instead of $0.27 \mathrm{M}_{\odot}$ of ${ }^{56} \mathrm{Ni}$, the model has a much narrower lightcurve than model DD4/27, as shown by the stars near $M_{V}=-19.01$ in the Figures 1 and 2 . In model M1 ${ }^{56} \mathrm{Ni}$ was mixed outward with overlying Si-group material. However the amount of material involved in the mixing was a small fraction of the total mass of ${ }^{56} \mathrm{Ni}$. Consequently the effectiveness of mixing in lowering the heating rate per gram and narrowing the lightcurve was small.

Interchanging Fe- with Si-group elements makes relatively little difference in the amount of energy released by burning a $\mathrm{C} / \mathrm{O}$ mixture; at most $6.2 \times 10^{17} \mathrm{erg} \mathrm{g}^{-1}$ is released burning to Si \& S versus $7.9 \times 10^{17} \mathrm{erg} \mathrm{g}^{-1}$ for burning to NSE. It is thus quite possible to imagine a series of explosions in which the $\mathrm{Si} / \mathrm{Fe}$ ratio varies more strongly than the specific kinetic energy. On the other hand, in most current models a greater production of ${ }^{56} \mathrm{Ni}$ is accompanied by a greater total amount of burning, leading to higher velocities. Model DD3 of Woosley \& Weaver (1991) produced $0.96 \mathrm{M}_{\odot}$ of ${ }^{56} \mathrm{Ni}$. It burned $10 \%$ more mass to the Si-group or above in total and has $10 \%$ greater kinetic energy than model DD4. In spite of its high ${ }^{56} \mathrm{Ni}$ mass, the lightcurve from this model is considerably narrower than that of model DD4/90 and is shown by the stars in Figures (1) and (2) at $M_{V}=-19.90$. The higher kinetic energy in this explosion resulted in a lower column depth, allowing decay $\gamma$-rays to escape more readily after peak. By 20 days, $\sim 6 \%$ of the decay energy in model DD4/90 escaped directly as $\gamma$-rays, increasing to $35 \%$ by day 40 . In model DD3, the escape fraction was more than double at 20 days, at $\sim 14 \%$, rising to $46 \%$ by day 40 . A shortened time to $\gamma$-ray transparency leads to a shorter rise-time (by 3.5 days), a narrower peak, and a greater decline rate in the lightcurve. The increased $\gamma$-ray escape was, however, more than compensated for by the shorter rise-time, and the peak magnitude is even brighter than the difference one might predict based solely on consideration of the difference in total ${ }^{56} \mathrm{Ni}$ mass. It is interesting to note that the $\Delta m_{15}(B)$ fitting procedure did not pick up this variation in lightcurve shape as it weights more heavily the decline rate after peak than the MCLS templates; the two models had very similar post-maximum declines. 


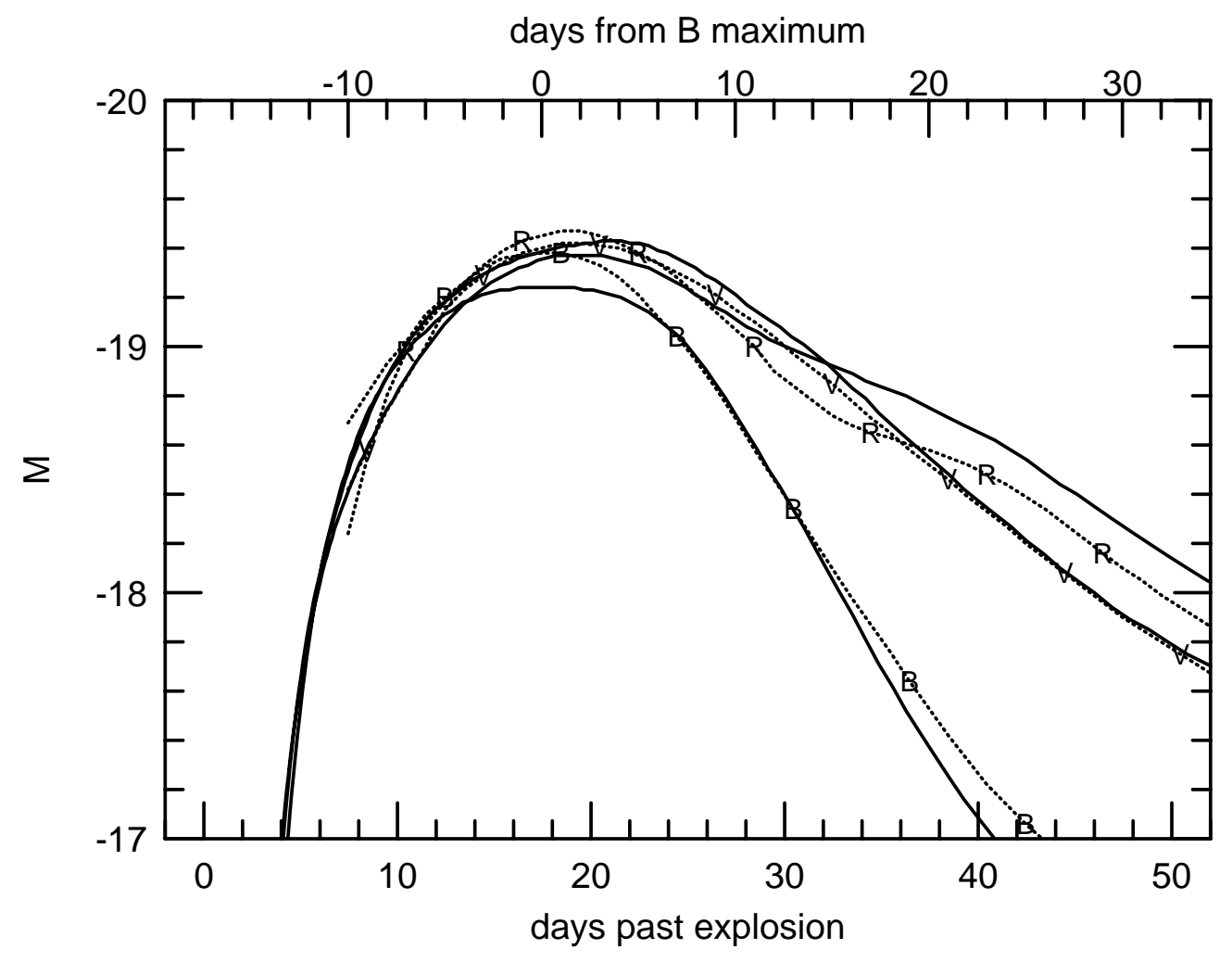

FIG. 4.- B, V, and R lightcurves of model DD4/55 and the best-fit MCLS templates on the interval $(-10,20)$ days relative to peak.

While far from a thorough investigation of all the variations one can imagine on explosions of $\mathrm{M}_{\mathrm{ch}}$ white dwarfs, the results presented above are suggestive. They show that it is possible to create a series of models which exhibit, to the best of our current calculation ability yet well within observational errors, the observed WLR.

It seems clear from the arguments of $\S 2$ and the results of these simple experiments that the ${ }^{56} \mathrm{Ni}$ yield and the $\gamma$-ray escape fraction play the determining roles in the relation between the lightcurve's width and peak luminosity. The models show a remarkable insensitivity to the distribution of radioactivity and to the composition of non-radioactive material, within limits set by achieving the appropriate explosion energies and acceptable nucleosynthesis.

The mass of ${ }^{56} \mathrm{Ni}$ sets the scale of the peak luminosity and the heating rate of the gas. The resulting temperature determines the ionization and hence the cooling rate of the gas. More ${ }^{56} \mathrm{Ni}$ leads to more heating, higher temperatures, less efficient cooling, and hence broader and brighter lightcurves.

The efficiency of $\gamma$-ray escape sets the evolution rate of the energy deposition. Models with higher overall velocities and/or with ${ }^{56} \mathrm{Ni}$ distributions extending to higher velocities exhibit larger $\gamma$-ray escape fractions at a given time. This increases the evolution rate of the lightcurve, with greater escape leading to narrower lightcurves. Models with higher ${ }^{56} \mathrm{Ni}$ yields tend to have their radioactivity distributed over a broader range in velocity, leading to larger escape fractions. This greater escape fraction acts to oppose the tendency for broader lightcurves resulting from higher temperatures, decreasing the slope of the WLR.

We have reproduced the observed relation in $\mathrm{V}$ by varying only the ${ }^{56} \mathrm{Ni}$ yield. One solution to reproducing the observed WLR is thus to produce an evolution scenario which leads to supernovæ with similar explosion energies yet differing ${ }^{56} \mathrm{Ni}$ yields. Because higher ${ }^{56} \mathrm{Ni}$ yields and higher velocities tend to produce opposing effects on the lightcurve, this solution is almost certainly not unique. A weak dependence of energy on ${ }^{56} \mathrm{Ni}$ yield will likely lead to acceptable lightcurves as well.

While to date the models which best fit lightcurves and spectra are all at the Chandrasekhar mass (with the possible exception of models for SN1991bg), the masses of all SN Ia explosions may not be identical. Varying the explosion mass primarily affects the $\gamma$-ray optical depth. As long as a given ${ }^{56} \mathrm{Ni}$ mass is accompanied by a column depth similar to those exhibited by the $\mathrm{M}_{\mathrm{ch}}$ models considered here, a variable-mass explosion scenario would not appreciably alter our conclusions. For sub- $\mathrm{M}_{\mathrm{ch}}$ model such as those of (Livne \& Arnett 1995; Woosley \& Weaver 1994), the ${ }^{56} \mathrm{Ni}$ produced by detonation in surface layers does not contribute appreciably to the optical lightcurve due to the low $\gamma$-ray optical depth. In such models, only the ${ }^{56} \mathrm{Ni}$ produced by carbon detonation in the core $\mathrm{C} / \mathrm{O}$ dwarf would affect the WLR.

The exact values of the slope and calibration of the WLR obtained in this work must be, to some extent, fortuitous. EP II showed that the conditions experienced by matter in SNe Ia are not consistent in detail with the assumption of LTE. The shape and colors of the lightcurve depend sensitively upon the average branching ratio from UV to OIR photons 


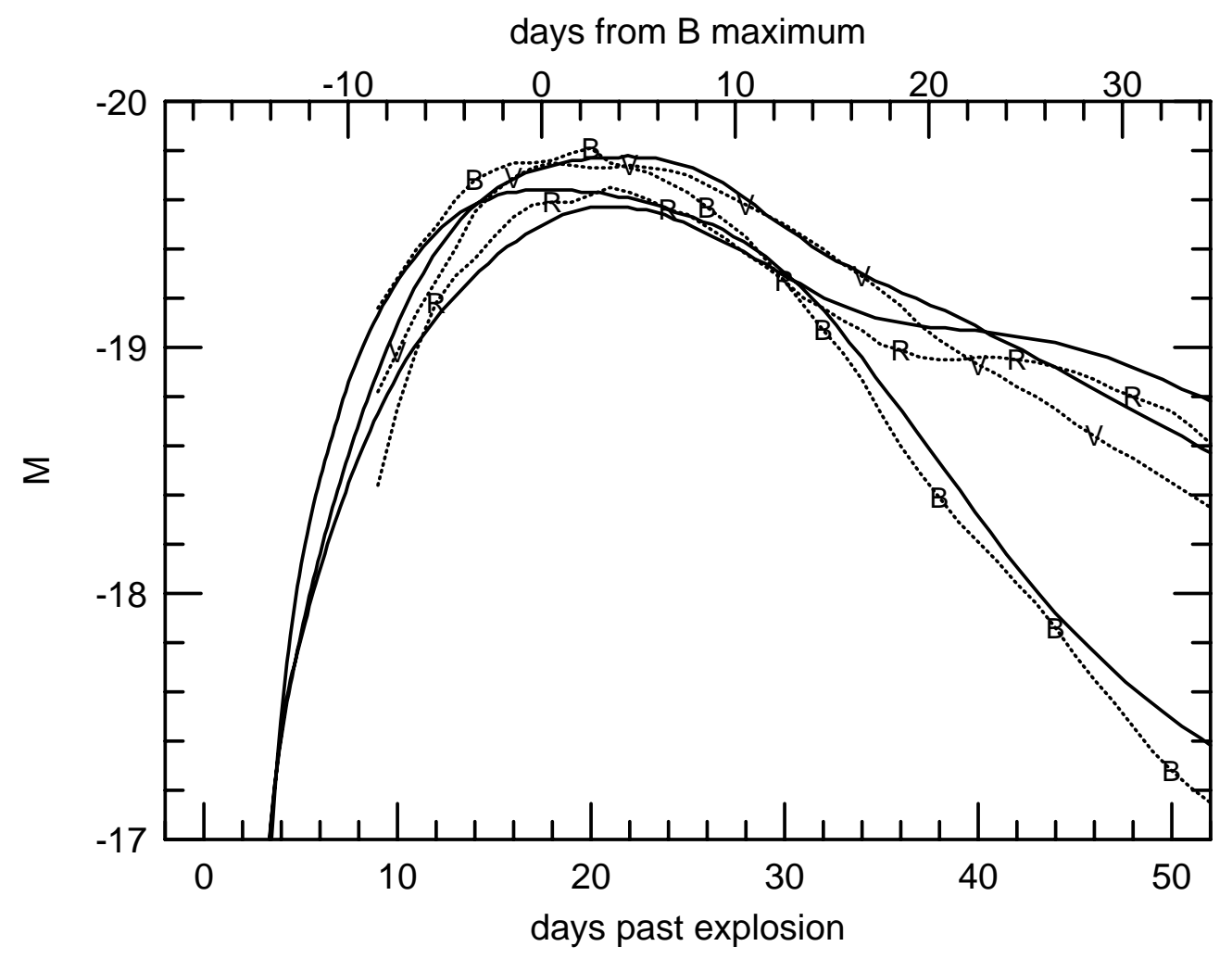

FIG. 5.- B, V, and R lightcurves of model DD4/90 and the best-fit MCLS templates on the interval $(-10,20)$ days relative to peak.

through a broad range in energies. While many strong lines are unlikely to be missing from our atomic models, all such models are seriously incomplete and the statistical properties of the line distributions and branching ratios are unlikely to be correct in detail. The basic physics, however, will not change significantly, and thus we have some confidence in the general trends the models indicate.

By fitting our models using the same procedures used to calibrate observed SNe Ia, we have demonstrated that the calibration is insensitive to fairly large changes in the underlying explosions. This suggests that the small differences in initial conditions which might arise from evolutionary effects between the local universe and that at $z \sim 1$ are unlikely to appreciably affect the supernova cosmology results. For example, mixing in model M1 leads to much less than 0.1 magnitude difference from the corresponding unmixed model. Even the unphysically large change in abundances in model $\mathrm{NiFe}$, strongly ruled out by chemical evolution considerations, leads to less than 0.2 magnitude departures from the MCLS and barely 0.3 magnitude departures from the $\Delta m_{15}(B)$ relations; allowable changes in relative iron-peak abundances would lead to insignificant effects.

Altering the specific kinetic energy, as in Model DD3, is the only way we see to achieve significant departures from the WLR. Increasing the kinetic energy by $10 \%$ was seen to lead to a much narrower lightcurve (at least when fit by the MCLS templates), primarily by more than doubling the $\gamma$-ray escape from $6 \%$ at peak in DD4/90 to $14 \%$ in DD3. This change is in the wrong direction, however. To account for the 0.3 magnitude deficit in brightness seen at large redshifts, a shift to lower explosion energy would be required. Halving the $\gamma$-ray escape from $6 \%$ at peak to $3 \%$ is a smaller fractional change in deposition and would lead to a smaller change in width. A future paper will explore these effects in greater detail.

We wish to thank M. Hamuy for many useful discussions and for performing the $\Delta m_{15}(B)$ fits to the lightcurves, and A. Riess for supplying his lightcurve templates in advance of publication. This work has been supported by the National Science Foundation (CAREER grant AST9501634), by the National Aeronautics and Space Administration (grant NAG 52798). This work was also performed under the auspices of the U.S. Department of Energy by University of California Lawrence Livermore National Laboratory under contract No. W-7405-Eng-48. Philip Pinto gratefully acknowledges support from the Research Corporation though a Cottrell Scholarship. 


\section{REFERENCES}

Arnett, W. D. 1982, ApJ, 253, 785

Castor, J., Abbott, D., \& Klein, R. 1975, ApJ, 195, 157

Eastman, R. G., \& Pinto, P. A. 1993, ApJ, 412, 731

Garnavich, P. M., et al. 1998, ApJ, 509, 74

Gibson, B. K., et al. 2000, ApJ, 529, 723

Hamuy, M., et al. 1996a, AJ, 112, 2408

Hamuy, M., Phillips, M. M., Suntzeff, N. B., Schommer, R. A., Maza, J., \& Aviles, R. 1996b, AJ, 112, 2391

Hamuy, M., Phillips, M. M., Suntzeff, N. B., Schommer, R. A., Maza, J., \& Aviles, R. 1996c, AJ, 112, 2398

Hamuy, M., Phillips, M. M., Suntzeff, N. B., Schommer, R. A., Maza, J., Smith, R. C., Lira, P., \& Aviles, R. 1996d, AJ, 112, 2438

Höflich, P. 1995, ApJ, 443, 89

Höflich, P., Khokhlov, A. M., Wheeler, J. C., Phillips, M. M., Suntzeff, N. B., \& Hamuy, M. 1996, ApJ, 472, L81

Iwamoto, K., Brachwitz, F., Nomoto, K. I., Kishimoto, N., Umeda, H., Hix, W. R., \& Thielemann, F. K. 1999, ApJS, 125, 439

Karp, A. H., Lasher, G., Chan, K. L., \& Salpeter, E. E. 1977, ApJ, 214,161

Kowal, C. T. 1968, AJ, 73, 1021

Livne, E., \& Arnett, D. 1995, ApJ, 452, 62

Mazzali, P.A., 2000, preprint.

Mazzali, P. A., \& Lucy, L. B. 1993, A\&A, 279, 447
Nugent, P., Baron, E., Branch, D., Fisher, A., \& Hauschildt, P. H. 1997, ApJ, 485, 812

Perlmutter, S., et al. 1999, ApJ, 517, 565

Perlmutter, S., Turner, M. S., \& White, M. 1999, Physical Review Letters, 83, 670

Perlmutter, S. e. a. 1997, ApJ, 483, 565

Phillips, M. M. 1993, BAAS, 182, 2907

Phillips, M. M., Lira, P., Suntzeff, N. B., Schommer, R. A., Hamuy, M., \& Maza, J. 1999, AJ, 118, 1766

Pinto, P., \& Eastman, R. 2000a, ApJ, 001, in press

Pinto, P. A., \& Eastman, R. G. 2000b, ApJ, 000, in press

Reinecke, M., Hillebrandt, W., \& Niemeyer, J. C. 1999, A\&A, 347, 739

Riess, A., et al. 1998, AJ, 116, 1009

Riess, A. G., Press, W. H., \& Kirshner, R. P. 1995, ApJ, 438, L17

Schmidt, B. P., et al. 1998, ApJ, 507, 46

Suntzeff, N. B. 1995, in IAU colloquium 145: Supernovae and Supernova Remnants, ed. R. McCray (Cambridge: Cambridge University Press)

Wagoner, R. V., Perez, C. A., \& Vasu, M. 1991, ApJ, 377, 639

Woosley, S. E., \& Weaver, T. A. 1991, in Les Houches, Session LIV, ed. J. Audouze, S. Bludman, R. Mochkovitch, \& J. Zinn-Justin (Elsevier Science Publishers)

Woosley, S. E., \& Weaver, T. A. 1994, ApJ, 423, 371 